Can. J. Math., Vol. XXII, No. 6, 1970, pp. 1208-1210

\title{
PERFECT MAPPINGS AND SPAGES OF COUNTABLE TYPE
}

\author{
J. E. VAUGHAN
}

In [1, p. 41, Theorem 3.10] Arhangel'skil proved that the perfect image of a completely regular space of countable type is of countable type, and he asked $[\mathbf{1}$, p. 60 , problem 4] if a similar result held for regular or Hausdorff spaces. In this paper, it is proved that the perfect image of a space of countable type is of countable type, provided that the image is Hausdorff or regular. An affirmative answer to both of Arhangel'skil's questions follows immediately from this. Arhangel'skir made use of the Stone-Čech compactification in the proof of his result, but the proofs below are of a different nature.

Let $X$ be a topological space and let $K \subset X$. A collection $\mathscr{U}$ of open sets is called a base at $K$ provided that for every open set $W \supset K$ there exists $U \in \mathscr{U}$ such that $K \subset U \subset W$. Clearly, we may assume that every member of $\mathscr{U}$ contains $K$. A set $K$ is said to be of countable character provided there is a countable base at $K$. A topological space is of countable type provided every compact subset is contained in a compact set of countable character. A surjection $f: X \rightarrow Y$ which is closed, continuous, and $f^{-1}(y)$ is compact for each $y \in Y$ is called a perfect map. It is well known that if $K$ is compact in $Y$ and $f$ is perfect, then $f^{-1}(K)$ is compact in $X$. Before giving the main results, we shall make several observations which will be useful later.

(I) Let $K$ be a compact set in a topological space, and let $\mathscr{U}$ be a base at $K$. If $\mathscr{F}$ is a filter base such that for every $U \in \mathscr{U}$ there exists an $F \in \mathscr{F}$ such that $F \subset U$, then $K \cap(\cap\{\bar{F}: F \in \mathscr{F}\}) \neq \emptyset$.

It follows from (I) that every compact set of countable character is contained in a compact $G_{\delta}$ of countable character. We may assume, therefore, that a space is of countable type provided that every compact set $H$ is contained in a compact set $K$ such that there is a countable base $\left\{K_{i}: i \in \mathbf{N}\right\}$ at $K$ with $K=\bigcap\left\{K_{i}: i \in \mathbf{N}\right\}$.

(II) Let $f: X \rightarrow Y$ be a continuous surjection, and let $S \subset X$. If there is a base at $S$ of the form $\left\{f^{-1}\left(V_{i}\right): i \in \mathbf{N}\right\}$ where $V_{i}$ is open in $Y$ for each $i \in \mathbf{N}$, then $f(S)$ is of countable character.

(III) Let $f: X \rightarrow Y$ be a closed continuous mapping and let $H \subset Y$. If $H$ is of countable character, then $f^{-1}(H)$ is of countable character.

Theorem 1. Let $X$ be a topological space of countable type, and let $f: X \rightarrow Y$ be a perfect map. If $Y$ is a Hausdorff space, then $Y$ is of countable type.

Received February 17, 1970. 
Proof. If $H$ is a compact set in $Y$, then $f^{-1}(H)$ is compact in $X$ because $f$ is perfect. Since $X$ is of countable type, $f^{-1}(H)$ is contained in a compact set $K$ of countable character. Let $\left\{K_{i}: i \in \mathbf{N}\right\}$ be a base at $K$ such that $\bigcap\left\{K_{i}: i \in \mathbf{N}\right\}=K$. Let $S_{1}$ be a saturated open set (i.e., $S_{1}=f^{-1}(V)$ for some $V$ which is open in $Y$ ) such that $f^{-1}(H) \subset S_{1} \subset K_{1}$. (This is possible since $f$ is a closed continuous map.) Assume that we have constructed open sets $S_{i}$ and $T_{i-1}$ for $1<i \leqq n$ which satisfy the following conditions:

(1) $S_{i}$ is a saturated open set for $i \leqq n$;

(2) $f^{-1}(H) \subset S_{i+1} \subset\left(T_{i} \cap K_{i+1} \cap S_{i}\right)$ for $1 \leqq i \leqq n-1$;

(3) $\left(K-S_{i}\right) \cap \bar{T}_{i}=\emptyset$ for $1 \leqq i \leqq n-1$.

We now construct $S_{n+1}$ and $T_{n}$ as follows. We have that $H \subset f\left(S_{n}\right)$ which is open, and $H$ and $f(K)-f\left(S_{n}\right)$ are disjoint compact sets. Since $Y$ is Hausdorff, there exist disjoint open sets $U$ and $V$ such that $H \subset U$ and $f(K)-f\left(S_{n}\right) \subset V$. Let $T_{n}=f^{-1}(U)$ and let $S_{n+1}$ be a saturated open set such that

$$
f^{-1}(H) \subset S_{n+1} \subset\left(T_{n} \cap K_{n+1} \cap S_{n}\right) .
$$

Now $\left(K-S_{n}\right) \cap \bar{T}_{n}=\emptyset$ since otherwise there exists $x \in\left(K-S_{n}\right) \cap \bar{T}_{n}$, which implies that $f(x) \in f\left(K-S_{n}\right)=f(K)-f\left(S_{n}\right)$ since $S_{n}$ is saturated, and thus $f(x) \in \bar{U} \cap V$ which is impossible. It follows that there exist collections $\left\{S_{i}: i \in \mathbf{N}\right\}$ and $\left\{T_{i}: i \in \mathbf{N}\right\}$ such that (1)-(3) are satisfied for all $n \in \mathbf{N}$. Let $S=\bigcap\left\{S_{i}: i \in \mathbf{N}\right\}$. We shall show that $S$ is compact. Since $S=\bigcap\left\{S_{i}: i \in \mathbf{N}\right\} \subset \cap\left\{K_{i}: i \in \mathbf{N}\right\}=K$, it suffices to show that $S$ is closed in $K$. If $p \in K$ and $p \notin S_{i}$ for some $i \in \mathbf{N}$, then $p \in K-S_{i}$. Hence by (2) and (3), $p \in X-\bar{T}_{i}$, which is open and misses $S_{i+1} \supset S$. Thus $S$ is closed. Now we have that $f(S)$ is a compact set containing $H$, and we can show it to be of countable character by showing that $\left\{S_{i}: i \in \mathbf{N}\right\}$ is a base at $S$ and applying (II). Suppose that $W$ is an open set containing $S$, but that $S_{i} \not \subset W$ for all $i \in \mathbf{N}$. Then $\left\{S_{i}-W: i \in \mathbf{N}\right\}$ is a filter base such that for each $i$, $S_{i}-W \subset K_{i}$. By (I), there exists at least one point

$$
p \in K \cap\left(\cap\left\{\overline{S_{i}-W}: i \in \mathbf{N}\right\}\right) .
$$

If, for some $i, p \notin S_{i}$, then $p \notin \bar{T}_{i} \supset \overline{S_{i+1}} \supset \overline{S_{i+1}-W}$. Hence

$$
p \in \cap\left\{S_{i}: i \in \mathbf{N}\right\}=S,
$$

but this is impossible since $p \notin W$. This completes the proof.

Theorem 2. Let $X$ be a topological space of countable type, and let $f: X \rightarrow Y$ be a perfect map. If $Y$ is a regular (not necessarily Hausdorf) space, then $Y$ is of countable type.

Proof. We proceed as in Theorem 1 , but construct a sequence $\left\{S_{i}: i \in \mathbf{N}\right\}$ of saturated open sets such that $f^{-1}(H) \subset S_{1} \subset K_{1}$ and

$$
f^{-1}(H) \subset S_{n+1} \subset \overline{S_{n+1}} \subset S_{n} \cap K_{n+1}
$$


for all $n \in \mathbf{N}$. This can be done since $f\left(S_{n}\right)$ is an open set containing the compact set $H$. Since $Y$ is regular, there exists an open set $U$ such that

$$
H \subset U \subset \bar{U} \subset f\left(S_{n}\right) .
$$

Let $S_{n+1}$ be a saturated open set such that

$$
f^{-1}(H) \subset S_{n+1} \subset\left(f^{-1}(U) \cap K_{n+1} \cap S_{n}\right) .
$$

Then $S=\bigcap\left\{S_{i}: i \in \mathbf{N}\right\}=\bigcap\left\{\bar{S}_{i}: i \in \mathbf{N}\right\} \subset \bigcap\left\{K_{i}: i \in \mathbf{N}\right\}=K$, and $f(S)$ is a compact set of countable character containing $H$.

Since Hausdorff and regular spaces are preserved by perfect maps, we have at once the following results.

Corollary 1. Let $X$ be a Hausdorff or a regular space of countable type, and let $f: X \rightarrow Y$ be a perfect map. Then $Y$ is of countable type.

COROLlary 2. Let $Y$ be a topological space and $\mathscr{F}$ a locally finite cover of $Y$ such that each $F \in \mathscr{F}$ is a closed Hausdorff (regular) subspace of countable type. Then $Y$ is a Hausdorff (regular) space of countable type.

Proof. Let $X=\sum\left\{F^{1}: F \in \mathscr{F}\right\}$ be the topological sum of the disjoint spaces $F^{1}$, where $F^{1}$ is homeomorphic to $F$ for each $F \in \mathscr{F}$. Then $X$ is a Hausdorff (regular) space of countable type, and the natural map $f: X \rightarrow Y$ (defined so that $\left.f\right|_{F^{1}}: F^{1} \rightarrow F$ is the given homeomorphism for each $F \in \mathscr{F}$ ) is a perfect map.

In conclusion, we remark that the inverse image under a perfect map of any space of countable type is of countable type. For let $f: X \rightarrow Y$ be a perfect map and let $Y$ be of countable type. If $H$ is compact in $X$, then $f(H)$ is compact in $Y$, and is contained in a compact set $K$ of countable character. Then $f^{-1}(K)$ is a compact set containing $H$, and is of countable character by (III).

The following question now arises naturally: Does Theorem 1 hold for an arbitrary space $Y$ ?

Added in proof. After this paper was accepted for publication the author learned that M. M. Čoban [Perfect mappings and spaces of countable type, Vestnik. Moskov. Univ. Ser. I Mat. Meh. 22 (1967), no. 6, 87-93] had proved that the perfect image of a Hausdorff space of countable type is itself a Hausdorff space of countable type. Hence, our Theorem 1 is a slight generalization of Čoban's result. Čoban also proved (what is our concluding remark) that the perfect inverse image of any space of countable type is of countable type.

\section{ReFERENCE}

1. A. V. Arhangel'skií, Bicompact sets and the topology of spaces, Trans. Moscow Math. Soc. 13 (1965), 1-62 (= Trudy Moskov. Mat. Obšč. 13 (1965), 3-55).

The University of North Carolina at Chapel Hill, Chapel Hill, North Carolina 